\title{
THE RELATIONSHIP BETWEEN THE BLOOD PRES- SURE AND THE TONIC REGULATION OF THE PIAL ARTERIES
}

\author{
BY
}

M. FOG

From the Medico-physiological Institute of the University of Copenhagen

(Received 29TH MaRch, 1938)

IN experiments on cats it was demonstrated that a decrease of blood pressure was followed by a dilatation of the pial arteries (Fog, 1934, 1937 ; Forbes, Nason, and Wortman, 1937) and that, conversely, a rise of arterial pressure caused a constriction of these vessels (Fog, 1938a). These vasomotor reactions were found to be independent of the procedures by which the fluctuations of pressure were produced. Stimulation of the sinus nerve or of the peripheral end of the cut vagus nerve or a diminution of blood volume had the same effect on the diameter of the pial arteries, provided that the same decrease in blood pressure occurred. Also the reactions during arterial hypertension were identical whether the rise in pressure was effected by the administration of adrenalin intravenously, stimulation of the splanchnic nerve, or increasing the blood volume. In this connection it is important to note that adrenalin was shown to have no direct chemical action upon the pial arteries of less than $50 \mu$ in diameter. On the other hand, vessels of larger than $100 \mu$ in diameter reacted to the hormone by distinct constriction, which, however, was not as great as that usually found in other vascular areas, e.g. the skin or the splanchnic area (Forbes and Wolff, 1928 ; Forbes, Finley, and Nason, 1933 ; Fog, 1934, $1938 b)$.

It was concluded from these observations that the muscular tonus of the pial arteries was controlled by the intravascular pressure, hypertension causing augmentation and hypotension diminution of the tone. The regulation, however, has certain limits. Thus, a sudden change of blood pressure may at first cause a "passive" reaction : the vessels dilate when a rise of tension starts abruptly or narrow at the beginning of a phase of hypotension. After 1 or 2 minutes these changes are usually followed by an "active" constriction or, in the second case, dilatation. If the rise of blood pressure is more than 100 per cent. above the normal level, dilatation of the arteries will almost certainly follow ; and if the level of blood pressure at the commencement is below $50 \mathrm{~mm}$. of mercury, active changes in the vessels do not occur. 
It appears from the experiments mentioned that the pial precapillary arterioles maintain at the normal level of arterial tension a minimum diameter, which cannot be reduced markedly by fluctuations of the intravascular pressure. If the systemic pressure descends below $100 \mathrm{~mm}$. of mercury there is a widening of the lumen proportional to the degree of hypotension ; during a subsequent rise to the normal level the artery will narrow to its original size. If the pressure is raised to $200-250 \mathrm{~mm}$. of mercury there will not be any further constriction. The tone of the arterial muscular wall is sufficiently augmented just to prevent distension, though excessive hypertension may, as mentioned above, lead to dilatation.

The existence within the cerebral vascular tree of a tonic regulation depending on the blood pressure was suggested as early as 1900 by Biedl and Reiner (1900). Since then the subject had apparently not been studied until the last few years. Finesinger and Putnam (1933) concluded that the influence of the systemic arterial tension upon the cerebral vessels was insignificant, but the results mentioned above point to an opposite conclusion. The question must be considered as of importance in view of the modern theories of general circulatory regulation.

According to W. R. Hess (1930), who has given an excellent survey of the subject, the actual "goal" of circulation is the proper distribution of the volume of blood according to the immediate needs of each part of the organism. Under the term "nutritive reflex" he includes all the neurogenic, chemical, and physical reactions by which the blood supply of a definite organ increases when this is required by its immediate functional needs. Not only are changes in the vascular bed of the organ itself and in the cardiac output active at this time but other circulatory areas take part in the regulation through a " collateral vasoconstriction." This causes a reduction of the volume flow in the resting organs, enabling the blood to be diverted to the organs in activity. Parallel to this adjustment to local needs the general blood pressure level is maintained or even raised, as during muscular exercise, by a specific reflex mechanism, which Hess has termed "Entlastungsreflex." It is activated by the tonus of the sinus and aortic nerves. The auto-regulation of the blood pressure under control of these "buffer nerves" is not only active during exercise but also meets the requirements of gravity and of heat regulation.

If the cerebral vessels were subject to a tonic regulation through the "buffer nerves" they should take part in the mechanism of the "Entlastungsreflex." In other words, there should be a cerebral vasoconstriction when the blood pressure tends to fall during the activity of other organs of the body or when there was a change from the horizontal to the erect posture.

Cobb and Finesinger (1932) concluded from experiments on the vasodilator nerves of the pial arteries that stimulation of the central cut end of the vagus nerve produced pial dilatation through a direct reflex from the vagus to the facial nerve centre. Ask-Upmark (1935) arrived at similar results when the sinus nerves were stimulated. In consequence, the "buffer nerves" were thought by these authors to exert the same indirect action upon the pial arterioles as upon other vascular areas. On the other hand, previous investi- 
gation (Fog, 1934, 1937, 1938a) has demonstrated that the response of the arteries on the surface of the brain to a fall or rise of blood pressure are identical whether all "buffer nerves" are cut or intact. Forbes, Nason, and Wortman (1937) found the vasomotor reactions to be in the nature of a dilatation when either the peripheral cut end of the vagus nerve or the sinus and aortic nerves were stimulated, an observation which indicated the " unspecific" nature of the response. Forbes, Nason, Cobb, and Wortman (1937) discovered that vasodilatation, following stimulation of the geniculate ganglion, was abolished after the local application of a solution of cocaine hydrochloride to the surface of the brain, whereas the reaction to a fall in blood pressure was preserved. In view of the last-mentioned results, it is justifiable to assume that the pial vessels do not take any part in a collateral vasoconstriction nor play an active part in the auto-regulation of the blood pressure, but that their tonus is decided by the level of the systemic blood pressure as controlled by the " buffer reflexes." This does not concern the possible action of metabolites produced during muscular or other exercise or the influence of a changing oxygen and carbon dioxide content of the blood.

The following experiments were planned to investigate the relationship between the blood pressure and the muscular tonus of the pial vessels more closely, and especially to study the influence of the activity of the sinus and aortic nerves in this respect.

\section{Method}

Cats, anæsthetised by butyl-bromallyl-malonylureid (Pernocton) were used. In all experiments artificial respiration was employed. The insertion of Forbes' cranial window and the measuring and recording of arterial diameters and of the blood pressure were accomplished as described in previous papers (Fog, 1934, 1937, 1938a). Variations of the blood pressure were produced by altering the blood volume. For this purpose a T-cannula was inserted into the abdominal aorta and connected to a special apparatus, shown in Fig. 1. A wide glass tube B (volume 200 c.c.), partly filled with defibrinated blood, connected above with a T-tube. One arm of the T-tube was connected to an oxygen tank $\mathrm{C}$ and the other to a vertical glass tube which dipped into mercury in the container $\mathrm{A}$. This tube could be lowered and raised to any level under the surface of the mercury during the experiments, so the length of the tube submerged in the mercury $(a)$ decided the pressure exerted upon the surface of the blood in the container B. Indirectly this pressure determined the systemic arterial tension of the experimental animal. When the tube in A was lowered blood would escape from B into the animal and the blood pressure be raised ; when the tube was raised a fall in systemic tension followed, as the blood ran from the animal into the apparatus.

In a number of cats the vagus, aortic, cervical sympathetic, and sinus nerves were divided on both sides. The total denervation of the carotid sinus was the most difficult part of the preparation. When the denervation was successful temporary compression of both common carotid arteries was not followed by a rise in general blood pressure ; if some branches of the sinus nerves were 
left, arterial hypertension resulted. Cats prepared in this way and successfully denervated will henceforth be termed " denervated " animals.

In all 32 cats were experimented on. In eight animals successful section of the vagus, cervical sympathetic, aortic, and sinus nerves was carried out. Changes of the blood pressure level was carried out 105 times and the reactions of the pial arteries were studied. In some animals several rises or falls of identical extent were produced from various initial levels of systemic tension. In others, a gradual fall was brought about from the normal level to 60 or

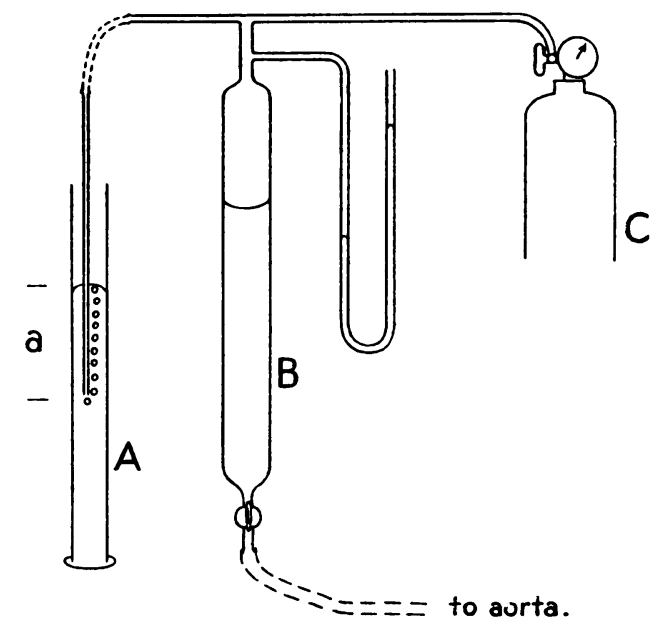

Fig. 1.-Apparatus for varying the blood pressure. A, mercury container. $a$, the height of mercury above the outlet for oxygen. B, blood container. C, oxygen tank. The apparatus was also used for compensating fluctuations of blood pressure in the experimental animal, see Fog $(1938 b)$.

$70 \mathrm{~mm}$. of mercury in the course of 30 minutes, whereupon the pressure was gradually raised to the original level or higher. Both types of experiments were carried out on " intact" as well as on " denervated" animals.

\section{Results}

The results are best illustrated by the study of some of the typical records. Fig. 2 represents the results in an "intact" animal. An arteriole under measurement presents a diameter of about $12-14 \mu$ with a normal blood pressure of $160 \mathrm{~mm}$. Hg. During the gradual reduction of the circulating blood volume, the blood pressure falls to about $80 \mathrm{~mm}$. $\mathrm{Hg}$, and the diameter of the pial vessel becomes doubled. A subsequent rise of blood pressure to $160 \mathrm{~mm}$. $\mathrm{Hg}$ brings the arteriole back to its original size. In Fig. 3 the results of a similar experiment performed on a denervated cat are presented and are seen to be essentially identical with those occurring in the "intact" animal (Fig. 2). In the denervated cat the rise of blood pressure, following the decrease, slightly exceeds the original level. In spite of this the artery did not contract further than to its original size. The responses of arteries larger than $100 \mu$ in diameter are best seen in another, intact, animal (Fig. 4). The variations of diameter are found to be in the same direction as in the smaller vessels, 
but occur apparently more slowly. It will be noted that an elevation of tension above $150 \mathrm{~mm}$. of mercury produces a narrowing of the lumen below its original diameter. Proportionally the vasomotor reactions in the larger vessels are far from being as pronounced as in the precapillary arterioles. In other animals the effect of sudden steplike rises of blood pressure from a low initial level

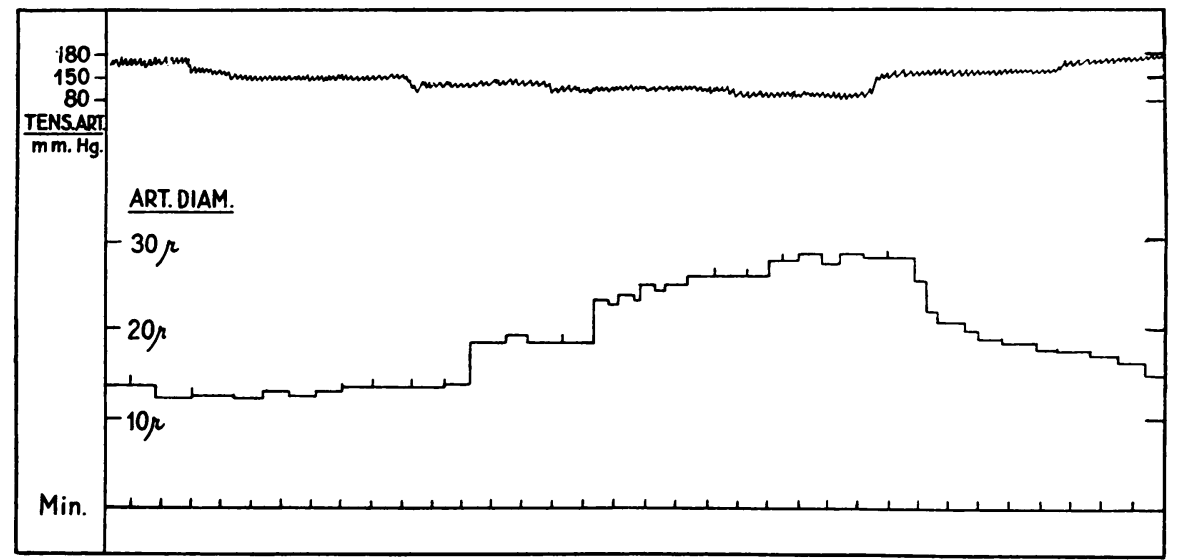

Fig. 2.-Reactions of a pial precapillary arteriole during decrease and increase of blood pressure, induced by variation of circulating blood volume. The vagi, sinus, aortic nerves, and the cervical sympathetic trunk intact.

was studied, of which Fig. 5 indicates the typical results. It is evident in this figure that the arteriole contracts most with the first rises of blood pressure.

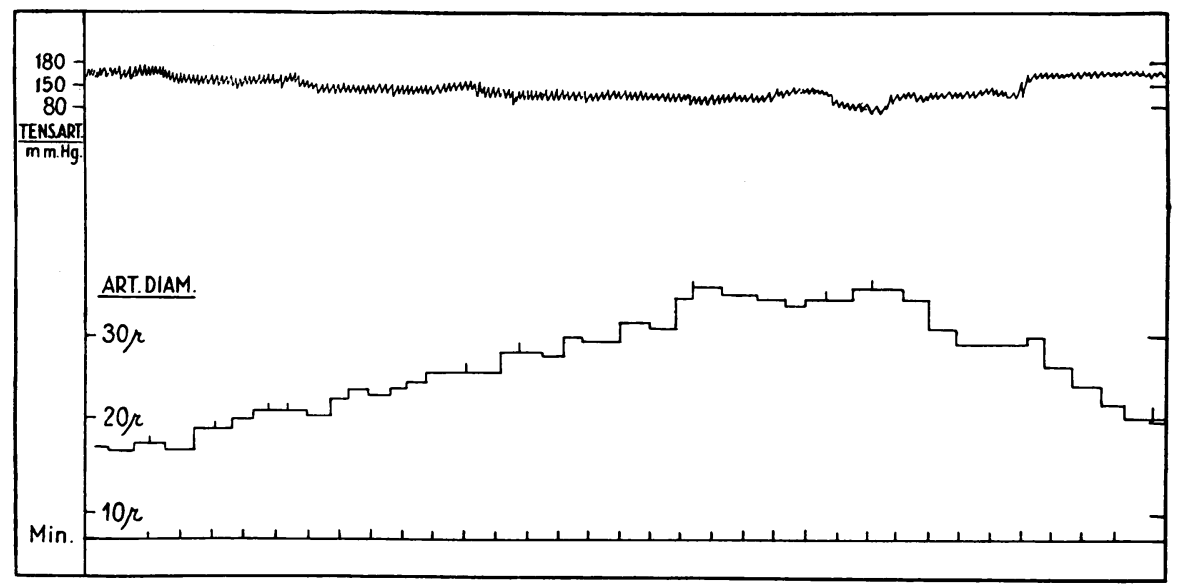

Fig. 3.-Reactions of a pial precapillary arteriole during decrease and increase of blood pressure. The vagi, sinus and aortic nerves, and the cervical sympathetic trunk severed.

These four records give the main features met with in the experiments. With variation of the intravascular pressure there was a change in diameter of the pial arteries. These results can only be interpreted as indicative of an active tonic regulation of the pial vessels. The tone is augmented when the blood pressure rises and is reduced when it falls. These reactions are inde- 
pendent of the presence of the cervical sympathetic and the " buffer nerves." They are common to the precapillary and the larger arterioles, but are most pronounced in the former. As long as the systemic tension is about the normal level there are only slight variations of lumen ; an active alteration of tone must, however, be assumed, since there is no dilatation during rises or con-

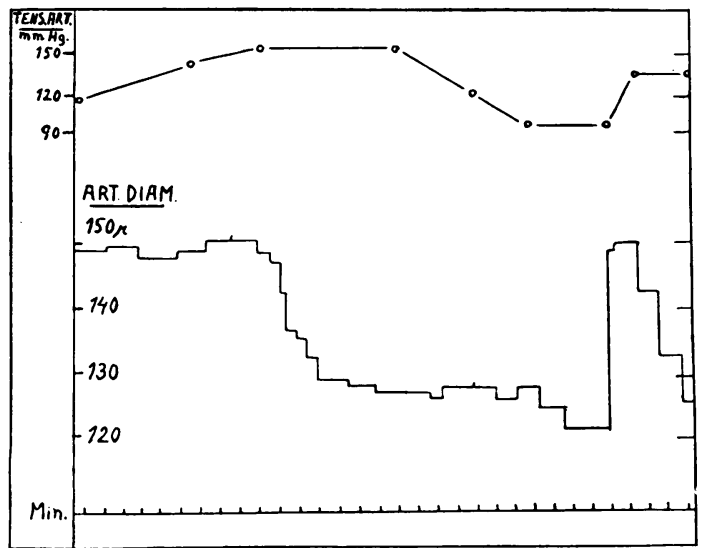

Fig. 4.-Reactions of a large pial artery during variation of blood pressure. "Intact"

tractions during falls in pressure. The smaller arterioles are not reduced below their normal size even if the pressure is raised to $180 \mathrm{~mm}$. of mercury, whereas the larger vessels in this case present some constriction. Obliterations were never seen. The level beneath which the tonic regulation entails obvious alteration of the diameter varies in different animals, but seems to be rather

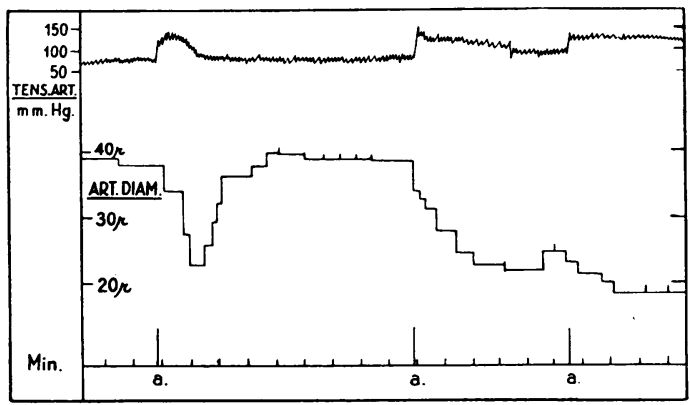

Fig. 5.-Reactions of a pial arteriole during sudden rises of blood pressure. "Intact" animal.

constant in any individual cat. Usually it was in the region of $110 \mathrm{~mm} . \mathrm{Hg}$, but occasionally as low as $90 \mathrm{~mm}$. $\mathrm{Hg}$.

Active responses were found to be pronounced in 24 cats. In another five animals the arterioles only presented slight alterations of diameter, not exceeding the error of the method of measurement $( \pm 2 \mu)$. As pointed out, this must be considered as a positive variation of vascular tone, which differs only quantitatively from that prevailing in cases where changes in diameter were 
observed. The vessels of the remaining three animals, however, seemed to follow quite passively the fluctuations of the intravascular pressure, since the vessels distended during rises and narrowed during falls of arterial tension. According to the notes of the experiment one of these cats was under unusually deep anæsthesia. The two others had been exposed to great losses of blood during the preparation for the experiment and at the start of the experiments their arterial pressure was below $40 \mathrm{~mm}$. $\mathrm{Hg}$. It is to be mentioned that another three cats had just as low a blood pressure at the beginning of the experiment, and at first reacted passively, but after a normal level had been maintained by the pressure apparatus for some time, their vasomotor responses became active ; thus apparently the normal tonic regulation may be regained.

In several experiments it was determined how much the volume of blood in the container varied during the alterations of the level of the blood pressure. Thus a quantitative expression was obtained of the changes of blood volume which were necessary to produce a certain rise or fall of the systemic tension. Though this aspect of the problem will not be examined here, it may be mentioned that the amount was considerably less in the denervated animals, a finding which illustrates the "buffer" action of the aortic and sinus nerves. When the average number of cubic centimetres of blood led to or from the animals per $100 \mathrm{~mm}$. of variations in blood pressure was computed from experiments on 18 cats it was found to be 100 to 115 c.c. in the intact as against 30 to 40 c.c. in the denervated cats. This only held for animals whose pial arteries were reacting actively. For the three cats with passive vasomotor responses the amount required was considerably larger, and in these cases it apparently made no difference whether denervation was performed or not.

\section{Comment}

The sensitiveness of the pial arteries to variations of the intravascular pressure is in the first place significant as an "emergency reaction," which comes into play when the auto-regulation of the systemic blood pressure fails, or, rather, is insufficient. It prevents, within certain limits, a rise of the capillary pressure during a rise in systemic blood pressure and thus protects the brain against injurious œdema. When the systemic blood pressure falls following hæmorrhage or shock this vascular reaction conduces to the maintenance of the cerebral blood flow by a reduction of the local peripheral vascular resistance.

Under various physiological conditions, moreover, these specific vasomotor responses are likely to be important factors, tending to keep the blood supply of the brain constant. The present series of experiments has confirmed the previous observation that the sinus and aortic nerves exert no influence upon the tonus of the pial arteries. Consequently, that vascular area will take no part in any of the functions which are elicited through the vasosensory nerves. This applies to the control of the blood pressure level, the adjustment of the circulation to local needs during exercise or other functional activity, and the effect of gravity during alterations of position. When, for example, the body 
is moved from the horizontal to the erect posture, there is a slight rise of the blood pressure as measured at the height of the heart. At the same time a decrease of tension will occur within the cranial vessels on account of the hydrostatic factor. If this decrease is not fully compensated for by the general rise of pressure, the pial vascular tonus will be reduced and may so entail a diminution of the local arterial resistance. It is the actual intravascular tension in the cranium itself, whether it results from the general "buffer action" or from hydrostatic factors or from a joint action of both, which determines the tonic state of the pial vascular walls.

It has been suggested by Leonard Hill (1896) in his fundamental papers on the cerebral circulation that the cerebral supply is largely dependent upon the systemic blood pressure, as the neurogenic vasomotor control of this area is only weakly developed. This thesis is maintained in principle by several authors to-day. The demonstration of a moderate sympathetic innervation of the larger pial arteries and of specific vasodilatory nerves in the fifth cranial nerve does not speak against it, since the functional influences of these are not yet established. The absence of control by the aortic and sinus nerves also seems rather to support Hill's thesis. In my opinion, however, the responses of the pial arteries to variations of the endovascular pressure are important factors in an autonomous regulation of the cerebral circulation, as they will secure a relative independence of the cerebral blood-flow during fluctuations of the systemic pressure. In opposition to the point of view advanced by Hill and others one might suggest that the blood supply of the brain is less dependent on the systemic pressure than that of other organs which possess a strong sympathetic innervation and are largely controlled by the tonus of the vasosensory nerves. The last-mentioned organs take part in the maintenance of a rather constant level of general tension and their blood flow varies in the service of this function. The vascular resistance of the pial vessels, on the other hand, is regulated inversely when it is necessary to maintain the cerebral flow unaltered. Even a small change of diameter has a marked effect on the flow.

It must be borne in mind that the blood flow is determined by the relation between the arterial tension and the peripheral resistance. The amount of blood which passes through the pial vessels under varying conditions cannot be measured. Possibly the dilatation of a pial vessel following a fall of general pressure may not be sufficient to keep the flow at its original amount; again, if the blood pressure rises beyond the normal level the absence of augmentation of vascular resistance may not prevent an excessive blood flow. Thus the reactions of the pial vessels may act only to modify the influence of variations of systemic blood pressure, i.e. to establish a partial compensating mechanism. Finesinger and Putnam (1933), who measured the blood flow in the internal carotid artery and so determined the supply of a larger area than the brain surface, stated that the flow was closely related to the pressure in that artery. Similar results were arrived at by Bouckaert and Heymans (1933) and others.

It has been claimed by Heymans and Bayless (1937) that morphiumPernocton-anæsthesia in dogs has a pronounced depressive action upon the 
vasomotor reactions. In the present series all cats were anæsthetised by Pernocton, but the results did not differ from previous experiments in which chloralosane and other drugs were used (Fog, 1934, 1937). The responses dealt with in this paper are evidently not abolished by anæsthesia. Further, it was mentioned that there was a pronounced difference in the amount of blood required to provoke a definite variation of systemic pressure when the vasosensory nerves were intact and when they were cut. This indicates that the function of the aortic and sinus nerves is preserved in cats under pure Pernocton anæsthesia.

It is interesting to compare the " physical " tonic regulation with its chemical correlate, viz. the effect exerted on the cerebral vessels by a varying tension of oxygen and carbon dioxide in the blood (Wolff, Lennox, and Allen, 1930). It

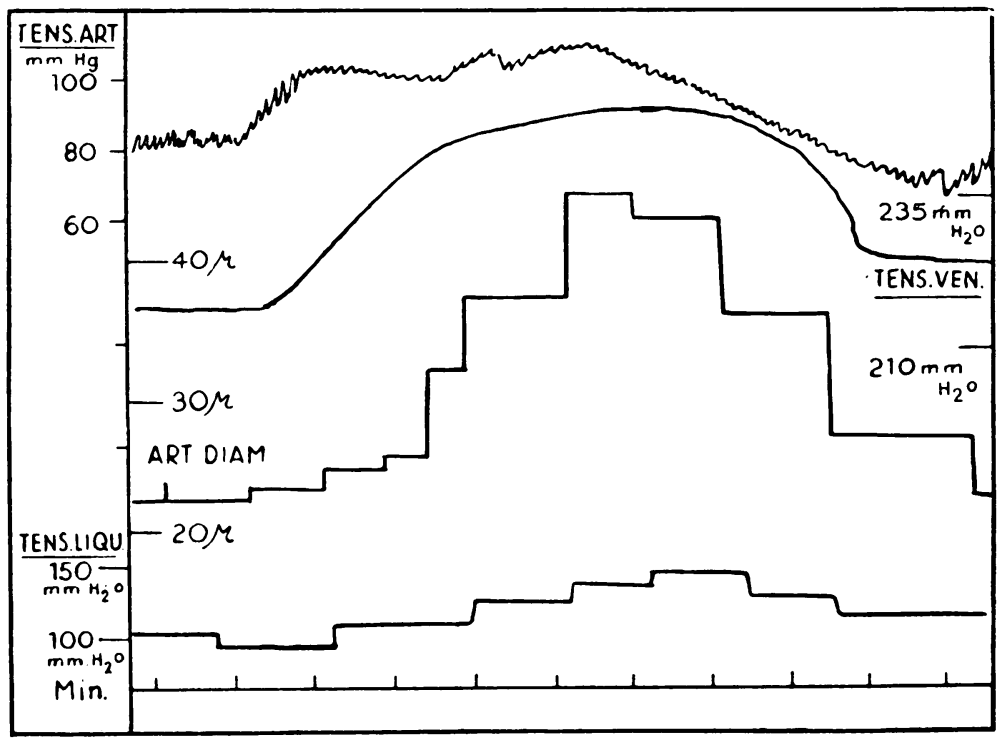

Fig. 6.-The reactions of cerebrospinal pressure, the diameter of a pial arteriole, the pressure of the external jugular vein and arterial pressure during asphyxia.

will suffice here to recall the pronounced dilatation of the pial arteries during asphyxia. In this condition there is a simultaneous rise of the systemic blood pressure ; thus the chemical and the physical regulation might be supposed to exert conflicting influences upon the cerebral vessels. It is evident from Fig. 6 that the action of the increased tension of carbon dioxide or the reduced oxygen tension or both together will be the predominant factors in such cases.

The nature of the tonic reactions which are elicited in the pial arteries and arterioles by variations of the intravascular pressure has not yet been established. If these reactions were only present with extreme changes of pressure, as when the blood pressure falls very low, they might be supposed to depend upon a coincident increase in carbon dioxide in the circulating blood, but as stated above the reactions come into play under physiological conditions, and further are present during normal respiration as well as during artificial ventila- 
tion. An extra-cranial reflex mechanism is unlikely, in view of their independence of the cervical sympathetic and the sinus and aortic nerves. Forbes, Nason, Cobb, and Wortman (1937), as already mentioned, found the responses to a fall of systemic pressure preserved after a local application of cocaine to the pia, whereas vasodilatation following stimulation of the geniculate ganglion was abolished. In all probability, therefore, the sensitiveness depends upon some local mechanism. Forbes, Nason, and Wortman (1937) call attention to the fact that pial arteries smaller than $800 \mu$ have no vasa vasorum. Elimination of metabolites produced locally by the arterial muscle cells takes place by diffusion, and even a slight retardation of the flow through the vessels may involve an increase of carbon dioxide within the tissue of the arterial wall, which again may cause a dilatation. On the other hand, it has been demonstrated that an augmentation of tonus follows upon hypertension up to 180 $200 \mathrm{~mm} . \mathrm{Hg}$; in these cases significant variations of elimination are not likely to take place. The same authors recall the suggestion made by Bayliss (1902, 1923) that the smooth muscle of the arterial wall reacts to distension by a contraction, and vice versa. Bayliss in the year 1923 regarded the question as undecided, but the present observations seem to support his original point of view. In that case the responses must be interpreted as a general property of the arteries of the body. Recent experiments of Porsaa (1938) have shown that the arteries of the retina react in a similar way to fluctuations of internal pressure as do the pial vessels.

\section{Summary}

In a series of experiments on 32 cats changes of systemic blood pressure were produced 105 times by alteration of the circulating blood volume. The pial vessels contracted with a rise in systemic pressure and dilated with a fall. The pial vascular responses were identical whether the sinus and aortic nerves, the cervical sympathetic trunk and the vagi nerves were intact or severed.

The reactions of the pial arteries resulting from change of the endovascular pressure indicate the presence of a vasomotor regulatory mechanism within the limits of the cerebral vascular tree. This mechanism assists in keeping the blood flow to the brain constant during various physiological and pathological conditions of the general circulation.

The nature of the specific mechanism is discussed and it is suggested that it depends on properties inherent in the smooth muscles of the arterial wall.

This investigation was aided by a grant from the Rockefeller Foundation.

\section{REFERENCES}

Ask-Upmark, C. E. F. (1935). “ "The Carotid Sinus and Cerebral Circulation.” Copenhagen. Acta Psychiat. Neurol., Suppl. 6.

Bayliss, W. M. (1902). J. Physiol., 28, 220.

(1923). The Vaso-Motor System. London.

Biedl, H., and Reiner, M. (1900). Pflüg. Archiv. ges. Physiol., 69, 158.

Bouckaert, J. J., and Heymans, C. (1933). J. Physiol., 79, 49.

Cobb, S., and Finesinger, J. (1932). Arch. Neurol. Psychiat., 28, 1,243.

Finesinger, J., and Putnam, T. J. (1933). Arch. Neurol. Psychiat., 30, 775. 
Fog, M. (1934). Om Piarrteriernes vasomotoriske Reaktioner. Danish Dissertation, Copenhagen.

(1937). Arch. Neurol. Psychiat., 37, 351.

(1938a). Ibid., in press.

$(1938 b)$. Ibid., in press.

Forbes, H. S., Finley, K. H., and Nason, G. I. (1933). Ibid., 30, 957.

Forbes, H. S., Nason, G. I., Cobb, S., and Wortman, R. C. (1937). Ibid., 37, 776. (1937). Ibid., 37, 334

Forbes, H. S., and Wolff, H. G. (1928). Ibid., 19, $1,057$.

Hess, W. R. (1930). Die Regulierung des Kreislaufes. Leipzig.

Heymans, C., and Bayless, F. (1937). Arch. int. Pharmacodyn., 56, 319.

Hill, L. (1896). The Physiology and Pathology of the Cerebral Circulation. London.

Porsaa, K. (1937). Unpublished data.

Wolff, H. G., and Lennox, W. G. (1930). Arch. Neurol. Psychiat., 23, 1,097. 